East African Medical Journal Vol. 80 No 4 April 2003

RISK FACTORS FOR STROKE AND USE OF ECHOCARDIOGRAPHY IN DIAGNOSIS: A REVIEW

A.J. Kinsara, FRCP, ABIM, Department of Medicine, King Khalid National Guard Hospital, Jeddah, P.O. Box 4409, Jeddah, 21491, Saudi Arabia

\title{
RISK FACTORS FOR STROKE AND USE OF ECHOCARDIOGRAPHY IN DIAGNOSIS: A REVIEW
}

\section{A.J. KINSARA}

\begin{abstract}
Background: Stroke is a prevalent disease in many communities. Cardiologists consultation and cardiac investigations are frequently requested to rule out cardiac source of embolic manifestations. The heart has been reported to account for $20 \%$ of ischaemic strokes in the Western community.

Objective: To review risk factors for stroke and the use of echocardiography in its diagnosis. Methods: Medline review was done for reported causes of stroke, these causes were divided into major and minor and were discussed briefly as general physician need to determine when and what to ask for.

Results: Some cardiac sources do have established roles in strokes but others have circumstantial and/or secondary roles.

Conclusion: While there exists evidence to recommend echocardiogram in stroke patients with heart disease, it is routinely needed in all patients with stroke. In some circumstances, echocardiography may enhance the diagnosis but not necessarily change management.
\end{abstract}

\section{INTRODUCTION}

Stroke is a major health problem and the socioeconomic consequences of the stroke are numerous and include financial, functional, social and psychological loss. It is the third leading cause of death in the world(1).

The role of cardiologist in stroke: Stroke is a condition that should be treated with the same enthusiasm as that used in managing a patient undergoing seizures. Patients who present within three hours of an episode should be considered for fibrinolytic therapy. Several risk factors are shared between ischaemic heart disease and ischaemic stroke and these have been well documented. Stroke has become an essential and integral part of Advanced Cardiac Life Support(2).

The true prevalence of the heart as source of embolism where intracardiac masses were identified, has been shown to be $4 \%$ by transthoracic echocardiogram (TTE) and $11 \%$ by transoesophageal echocardiogram (TEE) (3). This difference is due to close vicinity of TEE probe to the heart without barriers(4).

The heart has been reported to account for about $20 \%$ of ischaemic strokes(5). Other causes are atherosclerotic cerebrovascular disease such as carotid and intracranial vascular diseases which are responsible for about $20 \%$ of cases, penetrating arterial disease (lacunar) account for $25 \%$, cryptogenic stroke accounting for about $30 \%$ and the remaining $5 \%$ are attributed to other causes such as hypercoagulant state, vasculitis, carotid dissection and vasospasm(6-13). Consequently, the search for cardiac source of emboli is an important issue, which should be undertaken.
Major risk sources of emboli: The major risk sources of embolisation are those in which there is strong association with stroke due to their great potential for embolisation. They are regarded as minor when the association was noticed but not definite. A review of the recommended therapy based on American Heart Association/American College of Cardiology or American College of Chest Physicians with the reference to guidelines for clinical application of echocardiogram is suggested(14-16).

The major risk sources of embolisation are as follows:

Atrial fibrillation: Embolic rate reported varies according to the risk subgroup. In lone atrial fibrillation this risk is between 1-8\% per year(17). Anticoagulation (AC) is recommended with INR 2-3.

Mechanical valves: Embolisation risk for mechanical aortic valve is $1.5 \%$ per year. This is doubled in mechanical mitral valve(5). The guidelines recommend AC with INR 2.5-3.5 with addition of aspirin at a dose of $81 \mathrm{mg} / \mathrm{day}$.

Mitral stenosis: In patients with sinus rhythm and left atrial enlargement, embolic rate reported was $8-14 \%$. This is doubled with AF patients to about $31 \%$ and further increases to $65 \%$ when there is prior history of embolism $(5,18)$.

Intra-cardiac thrombosis: This occurs in the setting of post myocardial infarction particularly in the anterior cardiac area (19). AC with INR 2-3 as per American College of Cardiology/American Heart Association, however, American College of Chest Physician do not favour AC, but this was not in cases related to stroke

Recent myocardial infarction: Stroke rate of 1-2\% occurred annually (20). AC with INR 2-3 has been shown to decrease stroke rate. 
Dilated cardiomyopathy: This tends to have apical thrombi with an embolic rate of $4 \%$ per year(5). AC is controversial in this condition. However, observational data from SAVE and SOLVD STUDIES suggested beneficial effect with $\mathrm{AC}(21,22)$.

Aortic arch atheromatous plaques: The annual risk of emboli is $14-21 \%$. This is influenced by plaque morphology and size. Complex plaques more than $4 \mathrm{~mm}$ in diameter, mobile or ulcerated are more likely to embolise and had higher recurrent stroke rate (23-27). Options of treatment are $\mathrm{AC}$, the use of antiplatelet therapy or surgical resection $(24,25,28)$. Current evidence favours the use of AC with surgery being considered in highly selected cases. Although a significant decrease in stroke rate by surgical resection has been demonstrated in some reports $(29,30)$, overall surgical approach has been associated with a high post operative stroke rate(31).

Marantic endocarditis: This is defined as a nonbacterial vegetation that is smaller in comparison with bacterial vegetation, with variable echo density, less independent motion and usually located at the base of the leaflets. They have been described in malignancy and connective tissue diseases and may embolise in $14-90 \%$ of cases(32). The diagnosis depends on the clinical settings and treatment is directed towards the cause. Heparin had been suggested as therapeutic modality(33).

Infective endocarditis: In this condition a stroke rate of $12-40 \%$ per annum has been reported(34). Appropriate use of antimicrobial chemotherapeutic agents in addition to selective surgery, has been recommended.

Atrial myxoma: Stroke may occur in up to $25 \%$ of this condition. Surgery is the treatment of choice. Papillary fibroelastoma although benign may embolise leading to stroke(35).

Minor risk sources: Patent foramen ovale (PFO)stroke occurs in $2 \%$ per year following an initial episode of stroke when PFO is identified $(23,36)$. There is insufficient (no) evidence to recommend for or against any specific therapy for patent foramen ovale(3). PFO is common in normal people, hence stroke patients should be managed by $\mathrm{AC}$ and the opening closed by a device or surgery or venous interruption if they meet certain criteria like cases of recurrent cerebrovascular event or multiple infarcts on $\operatorname{MRI}(27,37,38)$. Another indication for closure is; PFO in which greater than 50 microbubles are seen in the left atrium on contrast TEE or stroke or transient ischaemic attack that are preceeded immediately by valsalva manoeuvre(27), Although PFO is common, paradoxical embolisation is rare, hence concurrent diagnosis of deep vein thrombosis or pulmonary embolism is thought to justify above therapeutic modalities $(6,13)$. The combination of PFO and atrial septal aneurysm has a substantial risk for recurrent stroke (39).

Mitral valve prolapse: Increased incidence of stroke is thought to be due to thickened, myxomatous valve, effect on left atrial size or increased incidence of atrial fibrillation(3,23). Aspirin is suggested for transient ischaemic attack and AC with INR 2-3 for stroke cases.

Mitral annular calcification: Its role is not well defined. Some authorities recommend AC, if the embolus is not calcified(15).

Ventricular aneurysm: Stagnation of blood is a good milieu for thrombus formation. Embolic rate is less than $1 \%$ per year(40).

Interatrial septal aneurysm: This is defined as bulging in the region of fossa ovalis more than $1.5 \mathrm{~cm}$ beyond the plane of atrial septum. The mechanism is not clear but in two thirds of the cases, patent foramen ovale was detected. Therapeutic measures are not defined yet, but antiplatelet therapy may be helpful $(23,39)$.

Calcified aortic stenosis: A common finding in the elderly. Studies are going on to determine its role in stroke and the effect of aspirin on its prevention(41).

Valvular strands: These are filamentous fibrin material that are thin and attached to aortic or mitral valve, also known as "Lambl's excrescences." It has become a common finding in clinical practice with the use of TEE but its role in stroke is debatable(42).

Spontaneous echocontrast: This is defined as swirling contrast due to slow blood flow in left atrium, also known as "smoke sign". it has been recognised more frequently with the advent of the use of TEE. However, echocardiogram settings may influence smoke detection, especially to the gain setting of the echo machine. It is often associated with atrial fibrillation. Embolic rate is not known. No specific therapy had been recommended(43).

Modalities of investigations that can be used: Three approaches of investigations can be used. Direct TEE examinations or TTE followed by TEE, if the former is not diagnostic or selective TTE or TEE. The decision as to which approach is appropriate in an individual case is dependent on a number of factors. First, before embarking on a specific test, it is important to elucidate the effect of test result on patient's management. Therefore, the echocardiogram should lead to identify for example, a condition requiring anticoagulation in a patient that will normally not be considered for such a therapy. In contrast, patients with atrial fibrillation or mechanical heart valve will require anticoagulation for their underlying problem, irrespective of the results of the test. On the other hand, patients with atrial fibrillation will not require echocardiogram for a diagnosis, since electrocardiogram will suffice. It is to be remembered that although TEE is much more sensitive for conditions such as left atrial thrombus and aortic arch atheroma(7-9), TEE carries a $0.2 \%$ risk of adverse effects (both major and minor) with $0.01 \%$ risk of death(10). The risk of fatality following TEE is ten times the risk of endoscopical examination. However, this has to be weighed against the risk of embolisation. If the source of embolism is identified, the risk of death increases considerably to $0-38 \%(11,12)$. Cost effectiveness of TEE has been confirmed in cases with heart diseases(13). 
The Canadian guidelines concluded that there is fair evidence to recommend echocardiography in patients with stroke and clinical evidence of cardiac disease by history, physical examination, electrocardiography or chest radiography. TEE is recommended as the preferred initial screening test, based on its sensitivity and costeffectiveness.

There is insufficient evidence to recommend for or against TEE in patients with normal results of TTE. There is insufficient evidence to recommend for or against routine echocardiography in patients (including young patients) without clinical cardiac disease. In contrast, the American guidelines recommend echo routinely for those who are below the age of 45 years (16). Routine echocardiography is not recommended for patients with clinical cardiac disease who have independent indications for or contraindications to anticoagulant therapy(5).

In conclusion, while there exists substatial evidence to recomend echocardiogram in stroke patients with heart disease, it is not routinely needed. These are conditions in which echocardiogram is not needed to make a diagnosis of the cause of stroke, which in others the performing echocardiogram may enhance diagnosis but not change the management of the patient.

\section{REFERENCES}

1. Bonita, R. Epidemiology of stroke. Lancet. 1992; 339:342344.

2. Guidelines 2000 for Cardiopulmonary and Emergency Cardiovascular Care. Circulation Supp. 2000; 102:204-211.

3. Anonymous. Cardiogenic brain embolism. The second report of the Cerebral Embolism Task Force. Arch Neurol.1989; 46:727-743.

4. Pearson, A.C., Labovitz, A.J., Tatineni, S. and Gomez, C.R. Superiority of transesophageal echocardiography in detecting cardiac source of embolism in patients with cerebral ischaemia of unceitain etiology. J. Amer. Coll. Cardiol. 1991;17:66-72.

5. Kapral, M., Silver, F. Canadaian Task Force and preventive Health Cares. Preventive Health Care, 1999-update echocardiograph for the detection of source of embolus inpatient will stroke. Can. Med. Ass. J. 1999; 161:989-996.

6. Blackshear, J. Transoephageal echocardiography in source of Embolism evaluation in the search for a better therapeutic rationale. Mayo Clin. Proc. 1999; 74:941-945.

7. Husain, A.M. and Alter, M. Transesophageal echocardiography in diagnosing cardioembolic stroke. Clin. Cardiol. 1995; 18:705-708.

8. Cha, R. Transesophageal echocardiography. New. Eng. J. Med. 1993; 90:680-684.

9. Chambers, J.B., de Belder, M.A. and Moore, D. Echocardiography in stroke and transient ischaemic attack. Heart. 1997; 78 Suppl 1:12-16.

10. Daniel, W.G., Erbel, R., Kasper, W. et al. Safety of transesophageal echocardiography. A multicenter survey of 10419 examinations. Circulation. 1991; 83:817-821.

11. Spirito, P., Bellotti, P., Chiarella, F., Domenicucci, S., Sementa, A. and Vecchio, C. Prognostic significance and natural history of left ventricular thrombi in patients with acute anterior myocardial infarction: a two-dimensional ecliocardiographic study. Circulation. 1985; 72:774-780.

12. Keren, A., Goldberg, S., Gottlieb, S. et al. Natural history of left ventricular thrombi: their appearance and resolution in the post hospitalization period of acute myocardial infarction. J. Amer. Coll. Cardiol. 1990; 15:790-800.

13. McNamara, R.L., Lima, J.A.G., Whelton, P.K. and Powe, N.R. Echocardiographic identification of cardiovascular sources of emboli to guide clinical management of stroke: a cost-effectiveness analysis. Ann. Intern. Med. 1997; 127:775787.

14. Task Force on Practice Guidelines (Committee on management of Patients with Valvular Heart Disease). ACC/ AHA guidelines for the management of patients with valvular heart disease: a report of the American College of Cardiology/ America Heart Association. J. Amer. Coll. Cardiol. 1998; 32:1486-1588.

15. Albers, G.W., Faston, J.D., Sacco, R.L. and Teal, P. Antithrombotic and thrombolytic therapy for Ischaemic stroke. Chest. 1998; 114(suppi 5): S383-S698.

16. American College of Cardiology/American Heart Association Task Force on assessment of diagnostic and therapeutic cardiovascular Procedures. ACC/AHA Guidelines for clinical application of echocardiography. Circulation. 1997;95:16861784.

17. Anonymous. Risk factors for stroke and efficacy of antithrombotic therapy in atrial fibrillation. Analysis of pooled data from five randomized controlled trials. Arch. Intern. Med. 1994; 154: 1449-1457.

18. Szekely, P. Systemic embolism and anticoagulant prophylaxis in rheumatic heart disease. Brit. Med. J. 1964; 1:209-212.

19. Vaitkus, P. and Barnathan, E. Embolic potential, prevention and management of mural thrombus complicating anterior myocardial infarction: a meta-analysis. J. Amer. Coll. Cardiol. 1993; 22:1004-1009.

20. Anonymous. Effect of long-term oral anticoagulant treatment on mortality and cardiovascular morbidity after myocardial infarction. Anticoagulants in the Secondary Prevention of Events in Coronary Thrombosis (ASPECT) Research Group. Lancet. 1994; 343:499-503.

21. Ezekowitz, M. Antithrombotics for left ventricular impairment. Lancet. 1998; 351:1904.

22. Al-khadra, A., Salem, D., Rand, W. et al. Warfarin anticoagulation and survival. a cohort analysis from the studies of left ventricular dysfunction. J. Am. Coll. Cardiol. 1998; 31:749-753.

23. Manning, W.J. Role of transesophageal echocardiography in the management of thromboembolic stroke.Amer. J. Cardiol. 1997; 80:D19-D28.

24. French Study of Aortic Plaques in Stroke Group. Atherosclerotic disease of the aortic arch as a risk factor for recurrent ischaemic stroke. N. Engl. J. Med. 1996; 334:1216-1221.

25. Mitusch, R., Doherty, C., Wucherpfennig, H. et al. Vascular events during follow-up in patients with aortic arch atherosclerosis. Stroke 1997; 28:36-39.

26. Witteman, J.C., Kannel, W.B., Wolf, P.A. et al. Aortic calcified plaques and cardiovascular disease (the Framingham Study). Amer. J. Cardiol. 1990; 66:1060-1064.

27. Devuyst, G., Bogousslavsky, J., Ruchat, P. et al. Prognosis after stroke followed by surgical closure of patent foramen ovale: a prospective follow-up study with brain MRI and simultaneous transesophageal and transcranial Doppler ultrasound. Neurology. 1996; 47:1162-1166. 
28. Ferrari, E., Vidal, I.R., Chevallier, T. and Baudouy, M. Atherosclerosis of the thoracic aorta and aortic debris; as a marker of poor prognosis: benefit of oral anticoagulants. J. Amer. Coll. Cardiol. 1999; 33:1317-1322.

29. Kronzon, I. and Tunick, P.A. Transesophageal echocardiography as a tool in the evaluation of patients with embolic disorders. Prog. Cardiovascular. Dis. 1993; 36:3960.

30. Laperche, T., Laurian, C., Roudaut, R. and Steg, P.G. Filiale Echocardiographie de la Societe Francaise de Cardiologie. Mobile thromboses of the aortic arch without aortic debris a transesophageal echocardiographic finding associated with unexplained arterial embolism. Circulation. 1997; 96:288294

31. Tunick, P.A. and I. Kronzon. Atheromas of the thoracic: aorta clinical and therapeutic update J. Amer. Coll. Cardiol. 2000; 35:545-554

32. Lopez, J.A., Ross, R.S., Fishbein, M.C. and Siegel, R.J. Nonbacterial thrombotic endocarditis: a review.Amer. Heart. J. 1987; 113:773-784.

33. Amarenco, P., Cohen, A., Tzourio, C. et al. Atherosclerotic disease of the aortic arch and the risk of Ischaemic stroke. N. EngI. J. Med. 1994; 331:1474-1479.

34. Sherman, D.G., Dyken, M.L., Gent, M., Harrison, M.J.G., Hail, R.G. and Mohr, J.P. Antithrombotic therapy for cerebrovascular disorders. An update. Chest. 1995; 108:S444S456.

35. Reynen, K. Cardiac myxomas . N. Engl. J. Med. 1995; 333:1610-1617.

36. Bogousslavsk, J., Garazi, S., Jeanrenaud, X., Aebischer, N.W. and Van Melle, G. Stroke recurrence in patients with patent foramen ovale: the Lausanne Study. Lausanne Stroke with Paradoxal Embolism Study Group. Neurology. 1996; 46:1301-1305.

37. Cujec, B., Mainra, R. and Johnson, D.H. Prevention of recurrent cerebral Ischaemic events in patients with patent foramen ovale and cryptogenic strokes or transient Ischaemic attacks. Can. J. Cardiol. 1999; 15:57-64.

38. Mas, J. and Zuber, M. French study group on patent foramen ovale and atrial septal aneurysm. Recurrent cerebrovascular events in patients with patent foramen ovale, atrail septal aneurysm or both and cryptogenic stroke or TIA . Amer. Heart. J. 1995; 130:1083-1088.

39. Mas, J.L., Avquizan, C., Lamy, C., Zuber, M., Cabanes, L., Derumeaux, G. and Coste, J., Patent Foramen Ovale and Atrial Septal Aneurysm Study Group. Recurrent cerebrovascular events associated with patent foramen ovale, atrial septal aneurysm, or both. N. Engl. J. Med. 2001; 345:1740-1746.

40. Lapeyre, A.C. III., Steele, P.M., Kazmier, F.J. et al. Systemic embolism in chronic left ventricular aneurysm: incidence and the role of anticoagulation. J. Amer. Coll. Cardiol. 1985; 6:534-538.

41. Otto, C.M., Lind, B.K., Kitzman, D.W., Gersh, W. and Siscovick, D.S. Association of aortic-valve sclerosis with cardiovascular mortality and morbidity in the elderly.

N. Engl. J. Med. 1999; 341:142-147.

42. Orsinelli, D.A. and Pearson, A.C. Detection of prosthetic valve strands by transesophageal echocardiography: clinical significance in patients with suspected cardiac source of embolism. J. Amer. Coll. Cardiol. 1995; 26:1713-1718.

43. Jones, E.F., Calafiore, P., McNeil, J.J. Tankin, A.M. and Donnan, G.A. Atrial fibrillation with left atrial spontaneous contrast detected by transesophageal echocardiography is a potent risk factor for stroke. Amer. J. Cardiol. 1996; 78:425-429. 